\title{
Three-Dimensional Modelling of the Middle-Ear Ossicular Chain Using a Commercial High-Resolution X-Ray CT Scanner
}

\author{
W. F. Decraemer, ${ }^{1}$ J. J. J. Dirckx, ${ }^{1}$ and W. R. J. Funnell ${ }^{2}$ \\ ${ }^{1}$ Biomedical Physics, University of Antwerp, RUCA, Antwerpen, Belgium \\ ${ }^{2}$ McGill University, Montréal, Quebec, Canada
}

Received: 17 June 2002; Accepted: 7 November 2002; Online publication: 10 February 2003

\begin{abstract}
The quantitative measurement of the three-dimensional (3-D) anatomy of the ear is of great importance in the making of teaching models and the design of mathematical models of parts of the ear, and also for the interpretation and presentation of experimental results. This article describes how we used virtual sections from a commercial high-resolution X-ray computed tomography (CT) scanner to make realistic 3-D anatomical models for various applications in our middle-ear research. The important problem of registration of the 3-D model within the experimental reference frame is discussed. The commercial X-ray CT apparatus is also compared with X-ray CT using synchrotron radiation, with magnetic resonance microscopy, with fluorescence optical sectioning, and with physical (histological) serial sections.
\end{abstract}

Keywords: three-dimensional, middle-ear, models, X-ray, computer, tomography

\section{INTRODUCTION}

The morphology of the middle ear is very complicated. In a narrow niche of complex shape within the temporal bone, the malleus, incus, and stapes, the tympanic membrane, the ligaments, and the muscles

Correspondence to: W. F. Decraemer - University of Antwerp - RUCA, Groenenborgerlaan - 171, Antwerpen, B-2020 - Belgium. Telephone: 3232180 430; fax: 3232180 318; email: wim.decraemer@ua.ac.be are intricately arranged in a 3-D configuration that continues to amaze even after many hours of observation. Classical textbooks illustrate this morphology using pictures and drawings with different viewing angles and different anatomical approaches, complemented with views from sections cutting through the temporal bone at different angles. Temporal bone preparations and physical 3-D scale models that can be opened or dismantled piece by piece are also used as reference or learning material. These models are based on typical ears and are therefore not well suited for the validation and interpretation of experimental results. For example, in middle-ear research many vibration measurements are performed on temporal bone preparations (e.g., at the tip of the malleus or at the footplate). Due to the great variation in anatomical size and shape, the orientation and position of the incident beam will likewise differ from experiment to experiment. When precise information is not available, it is hard to compare results from different experiments or different investigators.

The advent of high-resolution computed tomography (CT) made it possible to perform virtual instead of physical sectioning, and computer assistance facilitated the construction of reliable 3-D mathematical anatomical models. In this article, we demonstrate how CT with a commercial X-ray micro-CT scanner (SkyScan 1072) can be used to make detailed dedicated geometrical models for use in middle-ear mechanics studies. The commercial X-ray CT apparatus is also compared with X-ray CT using synchrotron radiation, with magnetic resonance microscopy, with fluorescence optical sectioning, and with physical serial sections. 
In particular, we show how micro-CT scanning was used in the framework of our recent study on the 3-D motion of the middle ear (Decraemer and Khanna 1999, 2000; Decraemer et al. 2000, 2002). Our experiments have two important parts: the measurement of the vibration of the ossicles and the measurement of the geometry of the ossicles. After completion of the vibration measurement part of the experiment, a high-resolution CT scan of the experimental temporal bone is made and, based on the resulting set of virtual sections, a 3-D reconstruction of the ossicular chain is made. We thus obtain a dedicated geometrical model of the ossicles in the specific temporal bone used during the experiment, and the experimentally determined motion parameters are used to animate this model. The procedure involves an important step, the registration of the model in the coordinate system used during the experiment, which is also discussed below.

The aforementioned registration procedure also makes it possible to increase the spatial resolution of a 3-D model by using two sequential scanning steps. This method is of general interest but we illustrate it with an example for the middle-ear ossicular chain. First, a low-spatial-resolution 3-D model is made based on a scan of the entire middle ear. Then, high-resolution scans of the isolated ossicles are obtained and the individual ossicle models are finally registered in the model of the entire structure.

It is not the goal of this article to thoroughly discuss all 3-D bio-imaging techniques in light of their application for temporal bone imaging, but we comment on the use of methods other than X-ray CT, based on our own experience. Similarly, we shall selectively cite papers on imaging of the ear when they fit in the present context.

\section{MATERIALS}

The choice of the material was guided by our current research on middle-ear mechanics. This encompasses studies on the middle ear of living anesthetized cat and on fresh (i.e., nonfixed) temporal bones of cat, gerbil, and human. Most often we first performed vibration measurements on the specimens and measured the geometry of the experimental temporal bones using high-resolution scans some days to several weeks later. In the case of the experiments on live cats, the animals were sacrificed at the end of the experiment and the temporal bones were removed. For cat the temporal bones were conserved either by letting them simply air dry or by storing them for a short time in a humid cloth or for a longer period in a fixative solution. Human specimens were stored in water with a few drops of antiseptic all the time they were used for experiments. Between experiments they normally started a few days postmortem and lasted at most a week - the temporal bones were kept in a refrigerator. After completion of the experimental sessions, the bones were stored in a fixative solution of about $4 \%$ formaldehyde. This article contains results for both cat and human temporal bones.

\section{METHOD}

\section{X-ray micro-CT scanner}

For the examples discussed in this article, we used scans made with the SkyScan 1072 X-ray CT scanner developed in cooperation with our university (Sasov and Van Dyck 1998). We briefly describe the measuring principle of this apparatus and comment especially on its applicability for middle-ear imaging.

A schematic drawing of the X-ray micro-CT scanner is shown in Figure 1. The object is mounted on a plate that can be rotated in the cone-shaped beam emanating from the X-ray point source. After alignment of the X-ray source and the center of the X-ray CCD camera along the "optical" axis of the apparatus, consecutive X-ray images are recorded while rotating the object by small angles (increment typically $0.90^{\circ}$ ). The pixel size can be varied between about 80 $\mu \mathrm{m}$ and somewhat less than $10 \mu \mathrm{m}$ by moving the object in the expanding beam either closer to the camera or closer to the source (pixel sizes for the smallest and largest magnification). During rotation all parts of the object must remain inside the scan volume, an imaginary cylinder with its axis determined by the rotation axis of the object holder and dimensions ranging from a radius of about $10 \mathrm{~mm}$ and a height of $20 \mathrm{~mm}$ to a radius of $1.25 \mathrm{~mm}$ and a height of $2.5 \mathrm{~mm}$. This condition determines the maximal magnification one can use for a given object. The accelerating voltage in the X-ray source can be chosen between 20 and $100 \mathrm{kV}$. An aluminium filter may be put in front of the source to truncate the spectrum of the X-ray beam. For our measurements beam intensity had to be high to keep recording time within reasonable limits (1-2 h) so the filter was not used and the accelerating voltage was always kept at its maximum. The contrast in each X-ray image depends on the X-ray absorption properties of the different parts of the objects. To get good visibility of embedded structures, the surrounding tissue should not absorb too well so that it does not act as a shield. A cone-beam back-projection algorithm calculates serial section images from the information at all angles for a horizontal line in the X-ray images. These can be stored with different resolutions ranging from $64 \times 64$ to $1024 \times 1024$ pixels. 


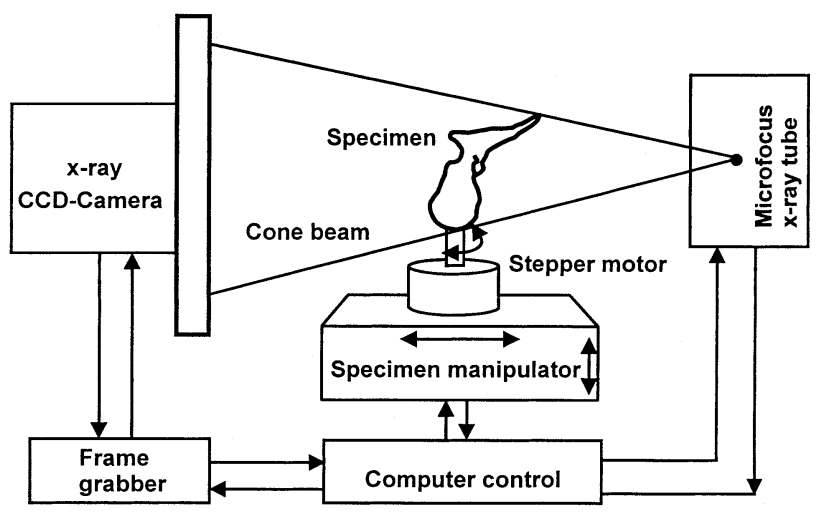

FIG. 1. Schematic illustrating the design and working principles of the SkyScan 1072 X-ray micro-CT scanner. The object is placed on a rotating table in a cone beam of the $X$-ray source. The camera records X-ray images for a set of equally spaced rotational positions. The computer controls all settings of the equipment, the rotational stepping, and image recording. A "back projection" algorithm then calculates a stack of virtual serial sections, cross-sections at the level of each line in the X-ray images perpendicular to the imaging plane.

The final result is a stack of serial images, each representing a horizontal section of the object (200400 slices typically) taken at regular intervals (an integer multiple of the slice thickness) in height. The slice thickness is equal to the pixel size in a section and hence depends also on the overall magnification.

\section{3-D model creation}

Whether we start with a set of serial sections obtained by physical sectioning or by virtual sectioning using computed tomography, the second step in the construction of a 3-D model is to identify the structure of interest (e.g., the malleus) in the sections where it is present. The object can be defined as the surface of an empty shell or as a solid volume. In the 2-D sectional image, the surface approach outlines the boundary of the structure as a contour, section by section. In a second step the object surface is constructed: A set of points on the surface ("nodes") and information about how these nodes are linked to their neighbors ("facet descriptors") are used to define the object. In a volumetric approach all pixels within the boundary of the structure are selected. This can be done section by section or by searching through the entire volumetric data stack.

Visualization of structures defined as surfaces is straightforward, and Web-oriented tools like virtual reality modeling language (VRML) browsers make it easy to share the 3-D models. For visualization of the volumetric data, one can either (1) explicitly derive the surface of the selected voxels as a second step, using a method like "marching cubes" (Lorensen and Cline 1987) or (2) use a direct volume-rendering method (e.g., Pfister et al. 2001). The latter approach may produce more realistic-looking images because the rendering uses the original gray levels or colors of the voxels and, to some extent, can avoid the explicit surface-identification problem; however, the rendering is computationally much more expensive. In any case, the explicit identification of surfaces is necessary if one wishes to use the 3-D model for, e.g., finiteelement modeling.

Essentially both methods have to detect the structure's boundary. There is no single best method for detecting the structure's boundary since the process depends strongly on the problem under study. For an isolated structure this is often a rather easy problem: a threshold applied to the gray-scale values of the images may be enough to clearly delimit the edges. For an embedded structure such as a middle-ear ossicle within a complete temporal bone, the task becomes much more difficult. Various, mostly semiautomated contouring procedures applying more or less sophisticated methods of edge detection between structures [e.g., simple threshold method, geometric deformable models (Mclnerney and Terzopoulus 1996), watershed method (Vincent and Soille 1991; Sijbers and Scheunders 1997), snakes (Xu and Prince 1997; Kass et al. 1987) can help to perform this task. Experience has taught us that most segmentation requires manual intervention, whichever segmentation procedure is chosen. This means that segmentation is always time-consuming. Any a priori, knowledge about the overall shape, position, and size of the object that one wants to segment can be useful; 3-D teaching models, pictures from anatomy books, histological images, and previous segmentations are often consulted during the segmentation. It can also be helpful to keep a set of direct X-ray images at selected rotation angles (say, every $20^{\circ}$ ) for consultation when the segmentation is not clear.

For most of the examples in this article, a commercial segmentation package (SURFdriver, http:// wwwsurfdriver.com/docs/default.html) that is based on a surface description was used. SURFdriver is distributed at reasonable cost jointly developed at the University of Hawaii (USA) and University of Alberta (CA) and offers a handy user interface. Its toolbox fulfilled most our demands. The segmentation was done mostly manually, although a semiautomatic fitting tool is provided. A limitation for us was that SURFdriver could not deal with "open contours", encountered when segmenting, say, the ear canal (an oblique section through the end of an open tube causes an open contour) or a thin structure such as the tympanic membrane (which shows up as a line in sections). Another program, Fie (Funnell 2002), was developed locally, in part to deal with this problem of open contours. Fie, which is available for free, provides (1) purely manual segmentation, aided by side 
views, display of lines from other slices, section-tosection copying and smoothing, etc., and (2) some primitive fitting algorithms based on intensity profiles computed perpendicular to initial lines drawn by the user. Fie was used for one example presented in the "Isolated cat stapes" section below.

A first-pass segmentation is followed by a second refinement during which the contours in consecutive sections are compared by displaying them simultaneously. The final quality of the segmentation process can best be judged in the next processing stage when a 3-D reconstruction of the object is made and displayed. This reconstruction step, or "surfacing," is incorporated in the SURFdriver program package or can be done with our locally developed Tr3 program (Funnell 2002). Other programs, such as the freeware program Nuages (http://www-sop.inria.fr/prisme/ logiciel/nuages.html.en), can also be used. Surfacing involves a triangulation of the points defining the different contours and can sometimes result in malformed surfaces if the shapes are convoluted; Tr3 permits interactive tuning of the triangulation control parameters (Funnell 1984). The rendering of the object surface follows the surfacing. Errors in the segmentation result in errors in the 3-D model. A lot of errors can be corrected by assuming that changes between contours of consecutive sections should occur gradually.

The 3-D model description, as a numbered set of points on the surface of the object and a list of number triplets defining triangular facets on the object surface by means of the node numbers, can be exported as text files from the surfacing programs that we used (Fie and SURFdriver). This makes it possible to use the data further to produce, for example, animations of chain motion, VRML visualizations, finite-element simulations, and other quantitative analyses.

\section{RESULTS}

In this section we give two segmentation and reconstruction examples, one of an isolated cat stapes and one of the ossicular chain in the human temporal bone with complete middle ear. The stapes is a small object that we could scan at high magnification and without hindrance of surrounding bone. In the second example, however, the ossicles were still embedded within the middle-ear cavity.

\section{Tomography of an isolated cat stapes}

The stapes was carefully dissected out of a left cat temporal bone that had been kept in a deep freezer over a period of about two years. The temporal bone
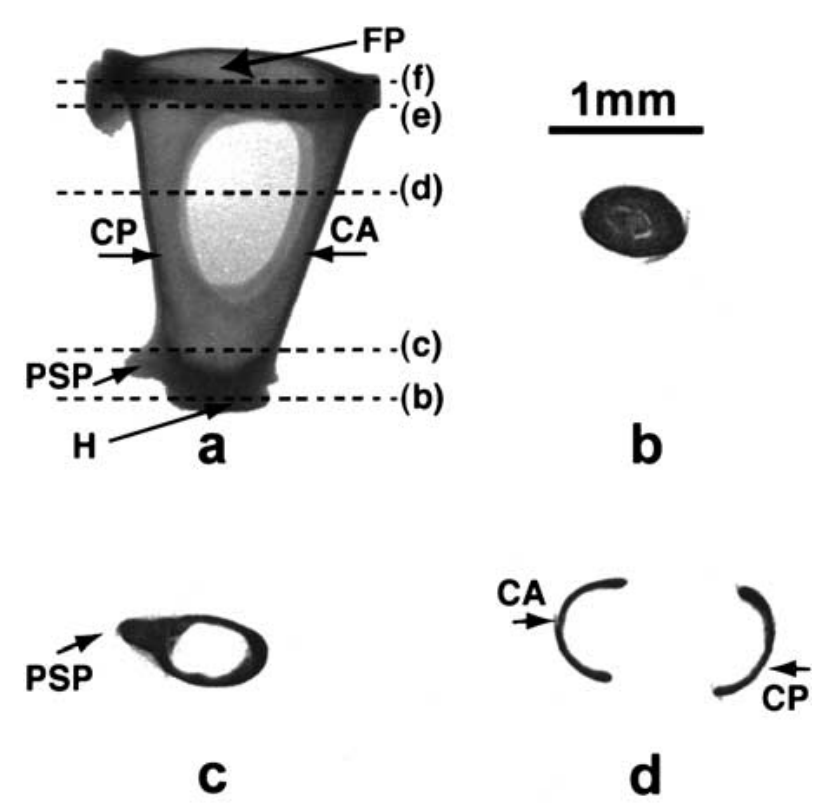

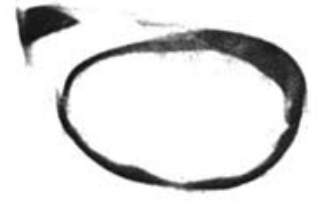

e

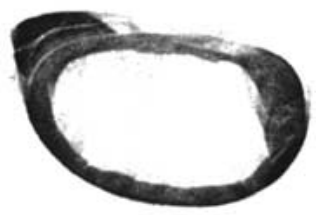

\section{f}

FIG. 2. CT scan of an isolated stapes of a cat. a. A direct X-ray image of the stapes mounted upside-down in the scanner. The crura are almost parallel to the image plane. The dark band around the edge reveals how thin the bone is. $\mathbf{b}-\mathbf{f}$. Cross-sections of the stapes perpendicular to the axis running from the head to the footplate at the levels indicated by the horizontal lines [annotated (b)-(f)] in a. Some anatomical landmarks are annotated as CA: crus anterior, CP: crus posterior, $\mathrm{H}$ : head of stapes, FP: footplate, PSP: posterior stapedial process. (Please refer to text for more details).

underwent no special treatment before it was frozen or after thawing for surgical preparation. The stapes was scanned using an early version of a SkyScan micro-CT scanner at $80 \mathrm{kV}$ and $100 \mu \mathrm{A}$ using a pixel and slice size of about $10 \mu \mathrm{m}$; the rotation step was $3^{\circ}$ and images were averaged over three frames. In Figure 2 we present a collage of images obtained from the scanner. Figure 2a shows one image out of the series of direct X-ray images of the stapes recorded during the scanning. The stapes was glued at its head to the object support of the scanner with the footplate upward and parallel to the support. The stapes is shown with the crura almost parallel to the image plane. Especially for isolated objects, the direct X-ray images provide a lot of information that can be very helpful during segmentation afterward. With this technique many of the well-known, detailed anatomical features 
of the ossicles can be observed. The thin dark gray band along most of the outer edge shows that the stapes is like a very thin hollow shell. The footplate can also be seen to be extremely thin. It has an outward convexity and is reinforced by an upstanding rim along its edge. The bony process of the posterior crus, where the stapedial tendon attaches, is clearly present. We marked it on Figure 2 as PSP (posterior stapedial process). This part, together with the top part of the stapes head $(\mathrm{H})$, appears darker because it is made of thicker bone than the rest of the stapes. The remaining lower part of the stapes head is completely hollowed out. The crura are formed by an almost perfect elliptical cutout. This specimen exhibits the usual asymmetry between anterior and posterior parts, e.g., we see that the posterior crus (CP) is somewhat broader than the anterior crus (CA) and slightly bent.

In Figure $2 b-f$ virtual sections are shown at different levels of the stapes as indicated on Figure 2a. Figure $2 b$ is a section through the solid part of the head. Figure 2c is a section through the hollow part of the head and through the posterior process to which the stapedius muscle tendon attaches (PSP). Figure $2 d$ shows the two semicircular sections of the two crura, while Figure 2e and $\mathrm{f}$ are sections taken at two levels of the footplate. The loose part at the upper left in Figure 2e and the extension at the upper left in Figure $2 \mathrm{f}$ are from a remaining fragment from the bone surrounding the annular ligament. The sections of this single object have distinct edges resulting in a straightforward segmentation. The segmentation of these images was done using our Fie program, and the high-resolution 3-D surfacing was done afterward using Tr3. The result is shown in Figure 3. Views from different viewing angles are given to show that the model reveals the fine details of this small structure. In Figure $3 a-d$, the stapes is seen in an upright position, rotated in steps about its long axis (from footplate center to center of the head), while in Figure 3e and f, the stapes is shown in top and bottom views, respectively. We eliminated the bony chip from the cavity wall during segmentation.

\section{Tomography of the ossicular chain within the human temporal bone}

The human temporal bone of this example is from an experiment on middle-ear ossicle vibrations; therefore, the middle-ear cavity was opened at the medial posterior side. To keep the entire temporal bone within the scanned volume of the CT scanner, the magnification had to be limited. For this tomography the cross-section pixel size and section-to-section distance was $21.0 \mu \mathrm{m}$. The rotation step was $0.45^{\circ}$ (we chose a small increment here since the specimen is

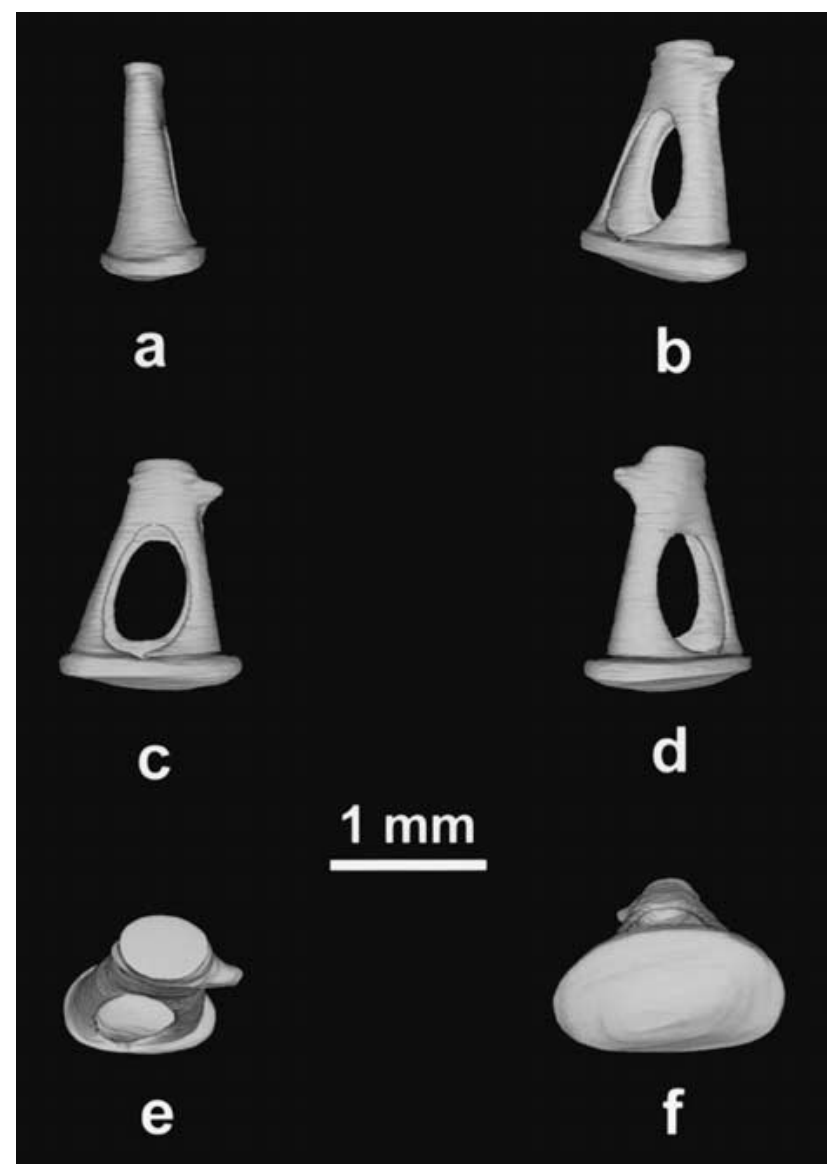

FIG. 3. Three-dimensional reconstructions of the stapes from six different viewing angles. a-d. The stapes is rotated in steps about the axis from footplate center to stapes head. e,f. Top and bottom views.

not an isolated object) and the scanner voltage and current were $80 \mathrm{kV}$ and $100 \mu \mathrm{A}$, respectively. Images were averaged over four frames.

Figure 4 is again a collection of a direct X-ray images (Fig. 4a) along with five reconstructed crosssections (Fig. 4b-f). Figure 4a does not teach us much in this case beyond the extent of the trimming of the temporal bone into a more or less cylindrical stub. We cannot distinguish structures inside the bony cylinder. A $z$-scale is added to Figure 4 a to indicate the $z$-level where the sections shown in panels b- $f$ were taken. A scale bar for dimensions in the sections is shown at the bottom of the figure. Panels b-d show sections taken at the level of the cochlea, whose turns can be observed in the center of the images. The section in Figure 4c shows the anterior crus (CA) and posterior crus (CP) as two crescent-shaped arches. In Figure $4 \mathrm{~d}$ the section cuts the head of the stapes which shows up as a small vertical ellipse $(\mathrm{H})$. In Figure $4 \mathrm{e}$ the section cuts through the bodies of the incus (I) and malleus (M). The last panel, Figure 4f, is a section at the level of the manubrium of the malleus (Man). Dark streaks in the cross-section 
images [e.g., at the right-hand side of the manubrium (Man) in Fig. 4f] are artifacts caused by a phenomenon known as beam hardening. During their passage through biological tissue, soft $\mathrm{X}$ rays are more easily absorbed than hard $\mathrm{X}$ rays (of higher energy) which causes a change in the energy spectrum of the beam known as "beam hardening." During interactive segmentation one can generally recognize these streaks clearly and ignore them when delineating boundaries.

The segmentation was done separately for each ossicle using SURFdriver. The outlining of the different structures is not as clear as it was for the isolated stapes shown in Figure 2, but it was nevertheless possible - in an iterative way as explained above to produce a very acceptable 3-D reconstruction (also done in SURFdriver) for each of the ossicles. Two views of each of the reconstructed ossicles are shown: for the malleus in Figure $5 \mathrm{a}$ and $\mathrm{b}$, for the incus in Figure $5 \mathrm{c}$ and $\mathrm{d}$, and for the stapes in Figure $5 \mathrm{e}$ and $\mathrm{f}$. For each ossicle we see that the overall shape is well reconstructed and that smaller details, e.g., the lateral process (LP) and anterior process of the malleus (AP), the lenticular process (Len P) of the incus with its fine stem, and the hollowed out and asymmetric aspect of the crura (CA and CP), are all present.

The three ossicles can also be displayed in relation to one another as all nodal coordinates are measured in the same reference frame. (The $x-y$ coordinates are defined along horizontal and vertical axes in each cross-section image and the $z$ coordinate is derived from the section number.) An example of the reconstruction of the three ossicles in their combined chain configuration viewed from eight different angles is shown in Figure 6. It is clear that a good 3-D model of the middle-ear ossicular chain can be obtained even with a set of CT sections of lower spatial resolution than that used for the isolated cat stapes. The total human temporal bone with its relatively large size and dense, solid bone encapsulating the middle-ear content was indeed at the edge of what our CT scanner was able to image. In Figure 6a, the ossicular chain is seen in an anterior-to-posterior view; in Figure 6d, it is rotated about the long axis of the stapes into a posterior-to-anterior view. Figure 6b$\mathrm{d}$ are intermediate positions for rotations about the same axis. Figure 6e shows a superior-to-inferior view while Figure $6 f$ gives a medial view. Figure $6 g$ has a posterior superior viewpoint, while Figure $6 \mathrm{~h}$ has a superior anterior viewpoint. Different aspects of the ossicular chain can be studied from these different views. For example, views with the stapes crura perpendicular to the image plane (Fig. 6a, d) and parallel to the image plane (Fig. 6c, e) are shown. In Figure $6 \mathrm{e}-\mathrm{h}$, the very special way the malleus and incus are joined can be observed. In Figure 6a, b, and
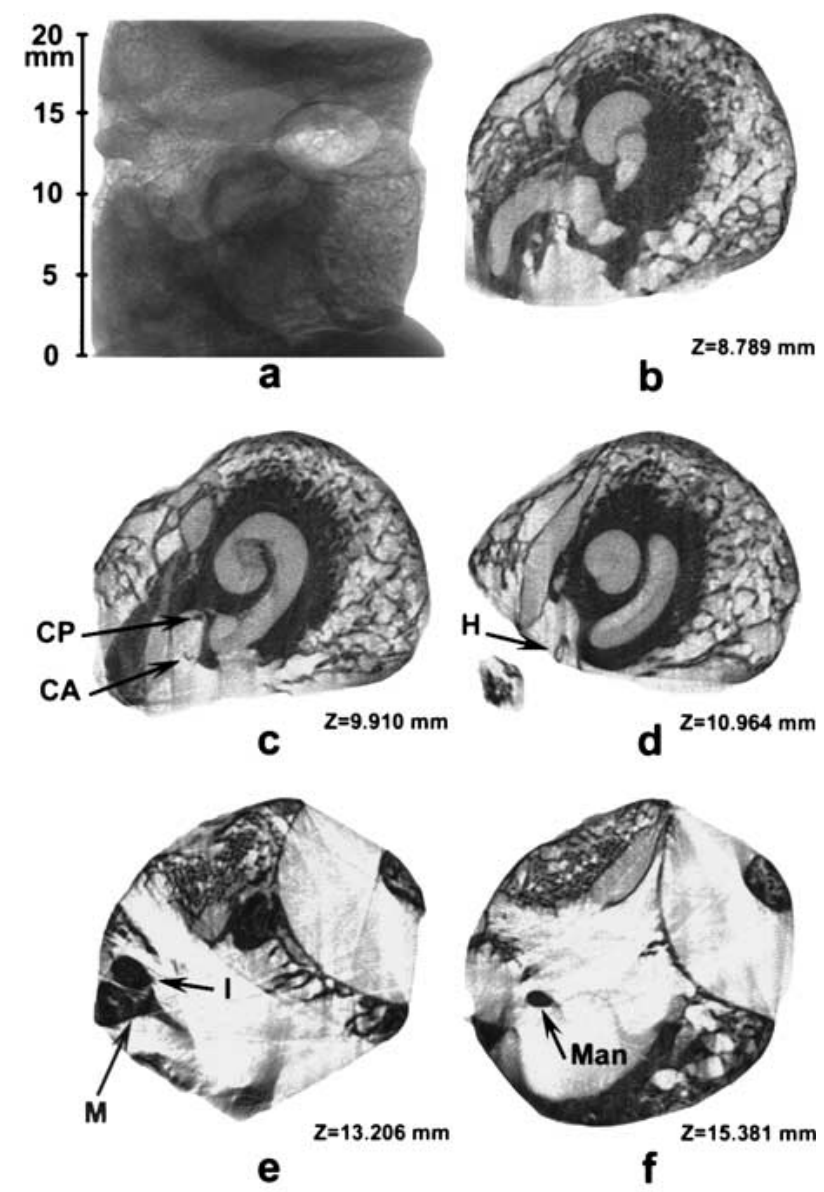

$10 \mathrm{~mm}$

FIG. 4. CT scan of an entire human temporal bone. a. Direct X-ray image of the temporal bone trimmed down into nearly a cylinder. $\mathbf{b}-$ f. Virtual sections at different heights of the specimen. The $z$ levels where the sections are taken on the scale of $\mathbf{a}$ are annotated in the panels b-f. Some landmarks are annotated: anterior crus (CA), posterior crus $(\mathrm{CP})$, head of the stapes $(\mathrm{H})$, the bodies of the incus $(\mathrm{I})$, and malleus $(M)$, manubrium of the malleus (Man).

$\mathrm{d}$, the direction perpendicular to the manubrium in the region of the umbo is seen to be close to the direction from the center of the footplate to the stapes head.

\section{Combining low- and high-resolution measure- ments}

In the previous examples we have shown that a single isolated ossicle can be reconstructed with high spatial detail but that this cannot be done for a total middleear chain in its original middle-ear housing. In cases where high resolution is required for a larger structure composed of different independent parts, such as the three-ossicle middle-ear chain, we have designed a three-step procedure. First, the entire structure is scanned at low resolution and a 3-D model is generated. Then the structure is physically 


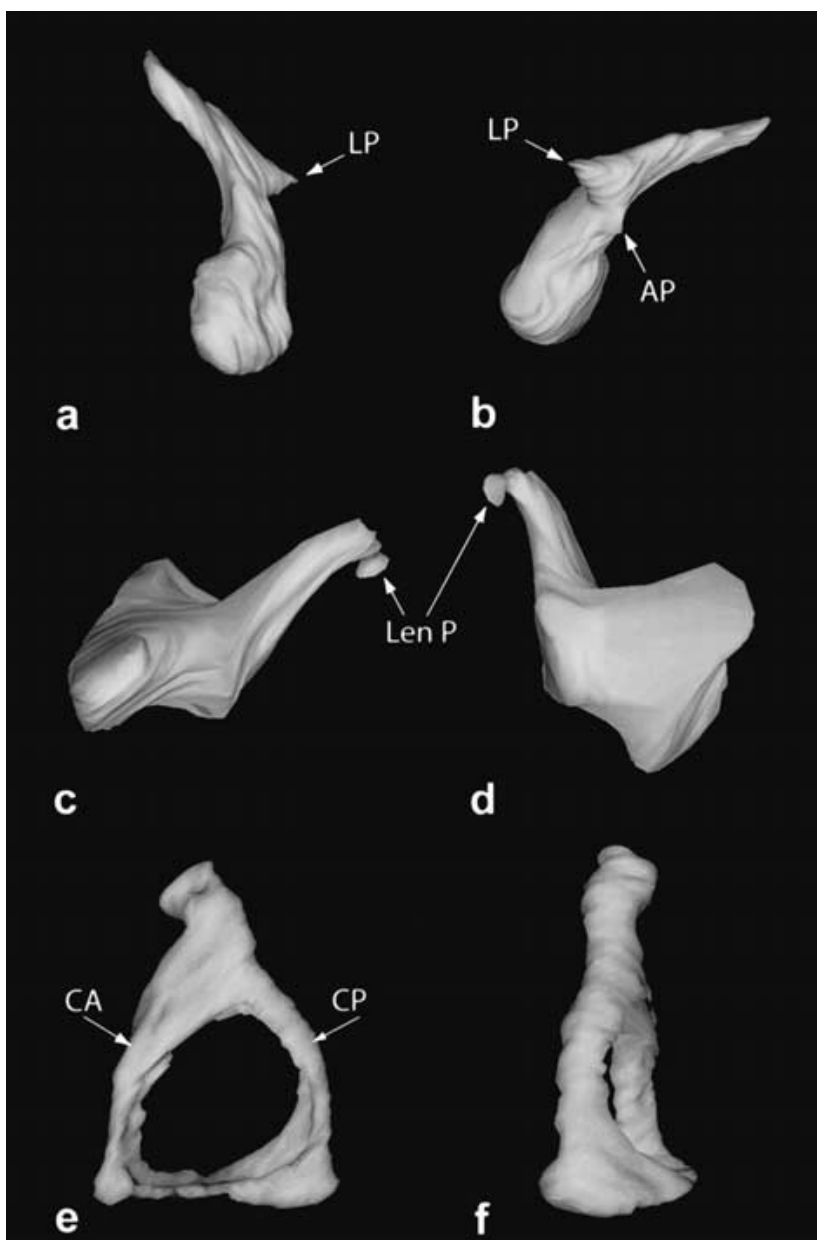

FIG. 5. The 3-D reconstructions of the three middle-ear ossicles after segmentation of the serial section stack of the human temporal bone shown in Figure 4. Two views for each ossicle are shown. a,b. The malleus. c,d. The incus. e,f. The stapes. Viewing angles are different for each ossicle and chosen to highlight some details: For the malleus, the lateral process (LP) and the anterior process are indicated, on the incus the lenticular process (Len P), and for the stapes the anterior (CA) and posterior crus.

dissected into its parts and these parts are scanned separately with high resolution. As a last step, the first low-resolution model is used to align ("register") the high-resolution single-ossicle models in the positions they occupied in the intact chain.

As an example we will show the construction of a highly detailed human ossicular chain.

Step 1: Low-resolution model of the human middle-ear chain. As a low-resolution model of the ossicular chain we shall use the total middle-ear chain model from the previous example (Fig. 6).

Step 2: High-resolution models for the isolated malleus, incus, and stapes. After further dissection the malleus, incus, and stapes were isolated and each of them was separately scanned using the highest magnification possible. The pixel width in the section images was $17.2 \mu \mathrm{m}$ for the malleus (scanned while the malleus

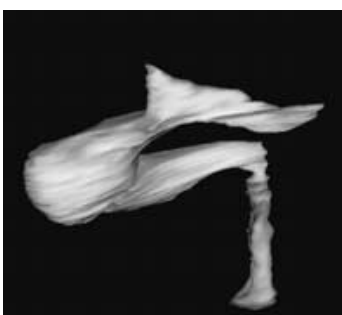

a

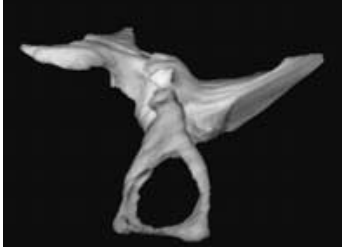

C

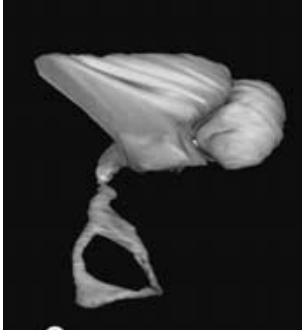

e

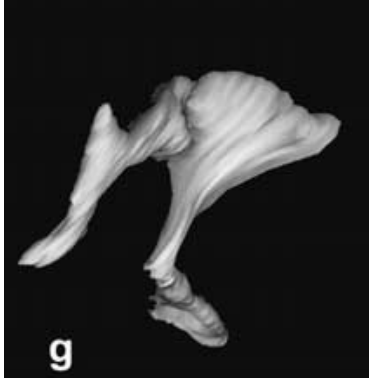

FIG. 6. The total middle-ear chain reconstruction combining the ossicle models shown in Figure 5. The malleus, incus, and stapes are shown in their normal anatomical relation to each other. Different viewpoints are used to illustrate this relation better as explained in the text.

was still attached to the tympanic membrane in the tympanic ring), $8.7 \mu \mathrm{m}$ for the incus, and $7.3 \mu \mathrm{m}$ for the stapes. For each ossicle we made a separate 3-D model. The three separate models for malleus, incus, and stapes are shown in 3-D views in Figure 7. It is clear that these 3-D models are of better quality than the corresponding models shown in Figure 5. The orientation of the ossicles is different for each ossicle in this figure as it was chosen to highlight fine details for each ossicle.

Step 3: Registration of the individual models in the total chain. To scan the total chain or the individual ossicles, the objects are positioned in the scanner so that the scanning volume is optimally filled. Therefore, 


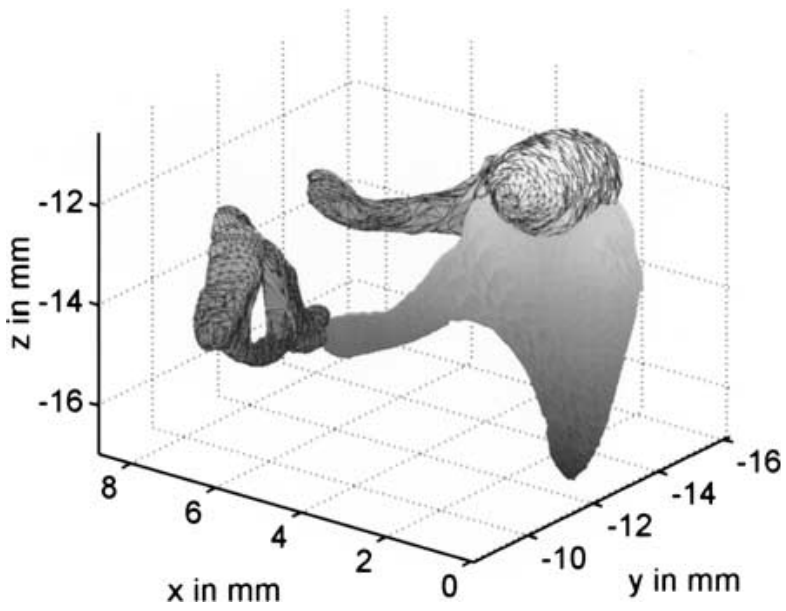

FIG. 8. The high-resolution 3-D model of the incus here is nicely replacing the low-resolution model in the total chain reconstruction shown in Figure 6. The position of the isolated incus model was calculated using an automatic registration procedure (ICP).

measure the coordinates of an extra set of about 100 "anatomical" points. This is a fast procedure that consists of focusing the interferometer at points spread across the entire visible part of the surface of the ossicle and automatically recording the coordinates (without vibration measurements).

The ICP procedure outlined above is then used to align the model with the combined set of observation points and anatomical points. As mentioned above, the initial guess for the transformation in the ICP procedure does not have to be very precise. As translations, it is sufficient to apply a translation specified as the difference in coordinates between two major landmark points (e.g., the center of the head of the stapes or the anterior process of the malleus) in the anatomical and model data sets. Then we manually rotate the model in an iterative way until the anatomical data points nearly coincide with the model's surface. A computer-screen plot serves as a check. (We performed this adjustment within Matlab, but any 3-D plotting program can be used.) It is advisable to temporarily shift the origin of both reference systems to the position of the landmark points. Rotating about a point on the object makes it much easier to visually follow the alignment of the model with the anatomical data set since the displacements due to the rotation remain small.

In Figure 9 we show an example of such a registration for the ossicles of a cat ear. In the upper panel, the chain model, after rough alignment, is shown together with the set of "anatomical" data points (black solid dots) measured to record the position of the chain in the reference system of the vibration experiment. In the lower panel, the chain model has been registered into the anatomical set. It can be seen that the anatomical data points fit the
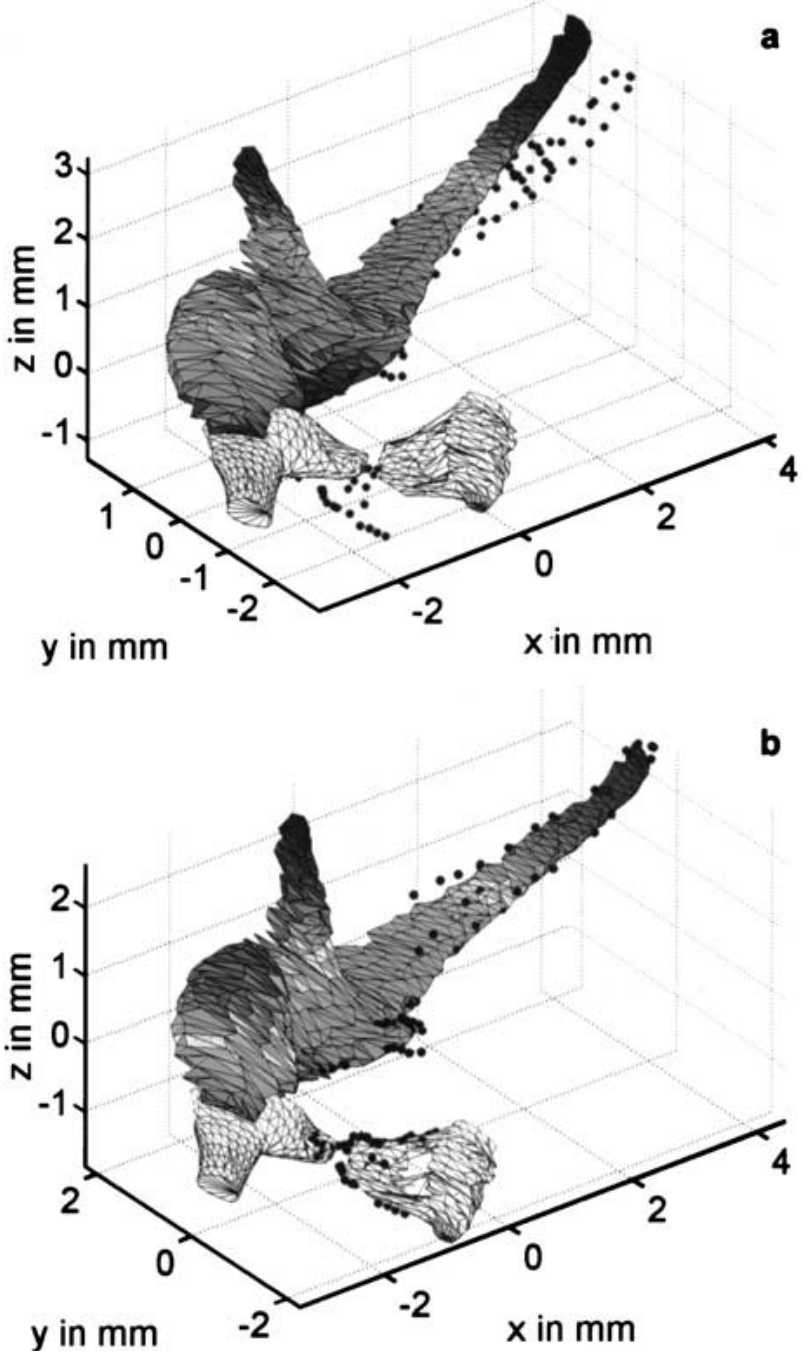

FIG. 9. The registration of the 3-D model of the total middle-ear chain in the set of anatomical points for a cat ear. a. The model is roughly aligned with the anatomical data set (solid dots). b. We see how the ICP procedure has now aligned the model to fit the anatomical data points closely.

model surface "like a glove." Small discrepancies are mainly due to the fact that soft tissue that was present during the experiment, e.g., at the edge between the manubrium and the tympanic membrane, at the head of the stapes, and at the lenticular process of the incus, is not imaged using X-ray CT and is therefore missing in the chain model.

\section{Combining measurements after a "loss of reference system"}

The registration procedure using an anatomical 3-D model of the structure as outlined above has another very useful application. When we want to make vibration measurements on a given ossicle from viewing angles beyond the $\pm 45^{\circ}$ range of our goniometer system, the object must be taken out of the holder for 
reorientation. As a consequence the reference system in which vibration components and coordinates of observation points are measured is lost. The same problem of "loss of coordinate system" can arise when, during an experiment, a manipulation to the object must be performed or when the object comes loose from the holder (e.g., the cement breaks). If an anatomical data set was registered before the coordinate change occurs and a new set of anatomical data points is recorded after the object is replaced in the holder, the 3-D chain model can be used to realign the experimental data sets before and after object replacement. First, we register the chain model with the first anatomical data set. Second, we register the second anatomical data set with the chain model as registered in the first step. This provides the transformation to bring the object back into the first experimental reference system. The object itself serves as a common spatial reference.

\section{DISCUSSION}

Anatomical data with high spatial resolution can be derived from sets of serial sections. These sections can be either ultrathin physical sections cut with a microtome or virtual sections produced by biomedical imaging tools such as X-ray, magnetic resonance, and positron emission scanners. (For an overview and introduction to various imaging techniques, see, e.g., (Robb 1995) Books on MRI include Haacke et al. 1999 and Kuperman 2000. Books on computed tomography include Herman 1980 and Kak and Slaney 1988.

\section{Techniques for imaging the temporal bone}

Here we discuss some techniques that have been used to study the anatomy of the middle ear. It is not our intention to go into the specifications of any measuring equipment since the underlying technology is constantly evolving, but we want to comment generally on strong and weak points of a few techniques as applied in middle-ear research. These comments are based on our own experience except for the use of synchrotron radiation.

Commercial X-ray micro-CT. The resolution of the virtual sections obtained with X-ray micro-CT varies with the magnification factor. With the highest magnification our scanner had a pixel size of about $8 \mu \mathrm{m}$. We showed that a fairly good model of the entire ossicular chain for cat and human can be made, but it is not without difficulties and a priori knowledge of the overall shape of the middle-ear ossicles is very useful to accomplish this task successfully. To best visualize the smallest structures of the middle-ear, one must minimize the thickness of the bone layer surrounding the middle-ear structures. This bone absorbs a lot of the X-radiation before it can reach the ossicles. In common experimental animals such as cat or gerbil (both often used as models in hearing research), the bone is rather thin and the temporal bones are quite small so that measurement of the total temporal bone is possible. For imaging the human temporal bone, much of bone surrounding the cavity has to be trimmed away. Therefore, CT scanning is not always "non destructive." When using the technique outlined above, combining low- and highresolution measurements of the larger structure and its smaller building blocks, respectively, the destruction of the specimen goes even further and the specimen as a whole is lost for future observation. In the inner ear the structures are an order-of-magnitude smaller than those in the middle ear, so that only the overall shapes of the scalæ but not the microstructures of the scala media can be imaged. These tissues are moreover quite soft so that they are hard to image with X-rays.

Non commercial X-ray micro-CT. Vogel (1998, 2000) performed outstanding measurements of the anatomy of the hearing organ using X-ray tomography. Complete human temporal bones (trimmed down to a cube of $20 \times 20 \times 20 \mathrm{~mm}$, but with intact middleand inner-ear structures) were measured using a microfocus X-ray tube scanner with very high beam intensity (250 mA compared with $100 \mu \mathrm{A}$ for the commercial SkyScan equipment) at a specialized research laboratory (Bundesanstalt fuer Materialpruefung, Berlin).

X-ray CT using synchrotron radiation. Smaller structures (incus, cochlea, and stapes) were measured by Vogel using synchrotron radiation (Hamburg Synchrotron Radiation Laboratory at the German Electron Synchrotron Facility, Hamburg, Germany). In Xray tubes (as in the SkyScan scanner and, the one in Berlin mentioned above), the radiation is generated as "bremsstrahlung." The radiation energy depends on the accelerating voltage of the electrons and on the specific characteristics of the target. Its spectrum shows peaks at energies that depend on the target material. Synchrotron radiation, however, is generated by the sudden change in direction of the electrons in a synchrotron caused by magnetic deflecting fields. The synchrotron radiation is extremely intense and broadband ("white spectrum") and emanates from an almost perfect source point. The very high intensity makes it possible to apply a narrow bandpass filter (monochromator) and obtain narrowband radiation that still has a high intensity. Vogel $(1998,2000)$ used radiation with energies of $24 \mathrm{keV}$ as a good compromise to image not only bone but also soft tissue, blood vessels, tendon, and nerves in the same preparation at high resolution. 
The high resolution that can be obtained with the two types of apparatus used by Vogel (about $2 \mu \mathrm{m}$ for the high-power microfocus tube, better than $1 \mu \mathrm{m}$ for the synchrotron radiation) causes the data sets to become very large so that an extra step of data reduction has to be performed. The results were used to make 3-D computer models and anatomically correct enlarged resin scale models that are ideal for teaching purposes. An obvious drawback of the technique is the limited accessibility of such measurement facilities.

Magnetic resonance imaging. We have also had the opportunity to analyze virtual sections of temporal bones (human, cat, gerbil, bat) obtained with magnetic resonance microscopy (MRM). The measurements were done at the Center for In Vivo Microscopy (Duke University Medical Center, Durham, NC, USA) on specimens specially prepared by M. Henson. A description of the technique and a good example of the measurements are given in Henson et al. (1994) and in a didactic presentation on their Web site: http://cbaweb2.med.unc.edu/ henson_mrm/pages/mrmfaq.html.

We can compare the quality of X-ray and MRM scans directly since one of the temporal bones used for vibration measurements on cat was scanned first with our X-ray micro-CT and later with the MRM apparatus. To use the highest MRM resolution (20 $\mu \mathrm{m}$ voxel size), the temporal bone was trimmed down to a $6 \times 6 \times 6$-mm cube to fit within the measurement space. MRM is based on the absorption and remission of radio-frequency electromagnetic energy; therefore, all surrounding bone and tissue is best removed beforehand. The system used is tuned to observe the hydrogen nucleus (proton nuclear magnetic resonance NMR) because this is abundantly present in the body. Therefore, hard bone material does not image well compared with soft biological tissue, so MRM can be complementary to X-ray CT.

To get an image of the ossicles, the middle ear cavity is filled with a contrast agent with high MR absorption (taking care not to introduce air bubbles) and the ossicles show up as islands within the liquidfilled cavity. At the cost of a nontrivial preparation and expertise in recording, data sets can be obtained with good definition of the middle ear cavity and ossicles.

The edge definition is comparable to or slightly better than that for X-ray micro-CT. (Note that MRI equipment is between five and ten times more expensive than an X-ray scanner for objects of comparable size.)

The position of the tympanic membrane is of great interest when studying the middle ear. For cat the visibility with MRM of the tympanic membrane is marginal. In one of the data sets that we used, sec- tions going through the superior regions showed the pars flaccida of the tympanic membrane from the anterior to the posterior edge, but of the pars tensa only scraps in the anterior and posterior parts could be seen in a few of the most superior sections. This corresponds to areas where the tympanic membrane is thickest (Kuypers et al. 2001). Thus, a complete tympanic membrane reconstruction could not be done. In another cat data set, the tympanic membrane was faintly visible everywhere. In gerbil, however, the surrounding bony capsule is much thinner, while in human the tympanic membrane itself is considerably thicker than that in cat, resulting in good visibility of the tympanic membrane in MRM scans of the gerbil and human temporal bones.

Other soft tissues of the middle ear, such as the tensor tympani and stapedius tendons, could in general be observed in the MRM data sets that we analyzed (human, cat, bat, and gerbil).

Orthogonal plane fluorescence optical sectioning. The orthogonal plane fluorescence optical sectioning technique (OPFOS, Voie 1996, 2001, 2002a, b; Voie and Spelman 1996; Voie et al. 1993) illuminates the specimen with a knife-blade-shaped beam and records images of the fluorescent structures in a direction orthogonal to the illuminating beam. The required setup is quite simple and, with proper choice of optical components, can achieve high spatial resolution. It was used for the imaging of the cochlea with great detail, revealing, e.g., the roundwindow membrane, basilar membrane, Reissner's membrane, cochlear aqueduct, and the microchannels in the modiolar wall (Voie 2002a). We have also created a 3-D reconstruction of the lenticular process of the guinea pig incus, with $2.6-\mu \mathrm{m}$ resolution in the plane of section (unpublished). The method has the disadvantage that the specimen must be decalcified, cleared, and impregnated with a fluorescent dye, and that local variation of the refractive index can occur and cause geometric distortion of the OPFOS images.

Physical serial sections. The traditional technique based on serial histological sections obtained with a real microtome has also been used in hearing research. The physical sectioning technique provides the greatest detail but making a good set of sections is very time consuming and requires a lot of skill. The specimen has to undergo decalcification and embedding before the actual risky process of sectioning and mounting of the slices on microscope glass plates. The slides are then subjected to a staining procedure. This is where physical sectioning excels, since the staining differentiates, e.g., collagen from bone or fat.

To study the 3-D anatomy of the sectioned specimen, segmentation has to be performed. In addition to the segmentation of each histological slide, there is 
also the need to align the section with the others in the stack. The process can be only partially automated; some manual intervention is always required and is generally very labor-intensive. The misalignment makes the segmentation itself more difficult as it disturbs the "continuity" in sequential slides. Histological sections are also prone to stretching, tearing, and other distortions, which degrades the quality of the 3-D reconstruction.

Such physical sections have been used to create middle-ear models for visualization (e.g., Nomura et al. 1989; Mason 2000). We used the technique to construct finite-element models of the middle ear (Funnell 1996; Funnell and Funnell 1988; Funnell et al. 1992) and to make teaching models (Warrick and Funnell 1998). Northrop (2001) made very complete 3-D models of the ear to study the aeration pattern of the middle ear in newborns. For practical reasons it is not feasible to make dedicated models for individual experimental animals with this technique.

\section{Application of 3-D models in hearing}

In the course of this article we have cited some examples where basic middle ear research benefits from the use of precise geometrical information about the ears under study. It is clear that in designing distributed models, such as finite-element models, the shapes and positions of the middle ear ossicles, tympanic membrane, ear canal, middle ear cavity, ligaments, muscles, and tendons are of major importance (e.g., Funnell et al. 1992). Other parameters, such as mechanical parameters like the (visco-) elasticity of all the components and the thickness of the tympanic membrane, should ideally be measured using the same ear that was used for the experimental measurements that we want to reproduce with the model. It is clear that this ideal situation cannot be realized, at least with current technology. Nevertheless, if an important aspect of the model, say the anatomical geometry, can be measured in the same ear that was previously used for experiments, this is a big step forward in the making of dedicated models. The CT data from X rays or MRI make this possible.

Knowledge of the geometry of the object under study is of great help for the kinematic and dynamic analysis of experimental results from motion studies, especially when 3-D aspects are studied. In middle-ear mechanics this means that, e.g., the anatomy of the tympanic membrane and middle-ear ossicles has to be precisely known. High-resolution CT techniques can be used to measure the middle-ear ossicle geometry. With MRM, soft tissue like the muscles and tympanic membrane can also be seen. It is expected that advances in technology will further improve the imaging of soft tissue. Sufficient resolution for soft and hard tissue will at first be achieved only for small objects, which is one of the reasons that studies on temporal bones (as opposed to in vivo studies) will persist at least into the near future.

\section{Geometrical accuracy}

An accuracy test of our X-ray scanner was performed using spheres of different diameters. No significant deviations from spherical shapes were observed. The deviations between the measured diameters and the sphere diameters were small (less than 2\%) for the magnifications used for the temporal bone measurements.

Different techniques require different methods of specimen preparation, as described above. Some preparation techniques can induce more or less important changes in the geometry of the specimen and thus induce errors in the anatomical measurements. The computerized X-ray scanner that we used does not require a special a priori treatment of the biological specimen, which is one of its strong points. The temporal bones that we scanned for our study were either deeply frozen for a while, simply dried (cat), or fixed in formaldehyde (human). The effects on the geometry (distortion of the ossicle position due to shrinking of the folds, ligaments, and muscle tendons) were not studied: Our primary goal was to get a good 3-D model for each single ossicle and then to register these models in the configuration that they had during the experiment and that was recorded in an anatomical data set. When absolute measurements of the entire temporal bone geometry are required, the use of fresh material is advisable.

\section{Publication of ear models on the Internet}

Physical models of the ear have long been used as study aids. The widespread availability of computer visualization has now made it possible to distribute virtual 3-D models on the Internet. A format that is very well suited for this purpose is VRML, but other formats or dedicated viewers could also be used [Warrick and Funnell (1998) give a VRML application example]. Normally the viewer program allows the model to be rotated and translated and zoomed in and out. These viewers often incorporate features that allow enveloping structures to be made transparent or allow sections to be made at arbitrarily chosen orientations ("reslicing" of the data stack) to facilitate observation. When such models can be freely downloaded and/or consulted, they will provide a valuable worldwide tool for medical students and researchers.

Some examples of models of the middle ear published on the Internet can be found on the following Web sites (in alphabetical order): 
- http://www.ruca.ua.ac.be/bimef/decraemer/ (Web site of W.F.S. Decraemer)

- http://funsan.biomed.mcgill.ca (Web site of W.R.J. Funnell)

- http://www.otovision.de/ (Web site of U. Vogel)

- http://cbaweb2.med.unc.edu/henson_mrm/ (Web site of O.W. Henson, Jr., and M.M. Henson)

\section{CONCLUSION}

We have presented applications of high-resolution Xray $\mathrm{CT}$ in the field of middle-ear research. We also discussed the applicability of other scanning techniques. Imaging instruments have been developed over the past few years, especially for studying small structures, and are still being improved upon.

Research-oriented CT scanners can be used to make 3-D reconstructions of the entire middle ear or of isolated middle-ear structures such as the ossicles. Imaging the entire human temporal bone, with its dense and thick bone, was the most difficult. Cat, rabbit, and gerbil ears have thinner bony capsules and the embedded structures are more clearly defined in the sections. Highly detailed 3-D models for isolated middle-ear ossicles can easily be made. MRI gives sharper edges of the ossicles and, in some preparations, even the thin soft structure of the tympanic membrane is also visible. Tendons and middle-ear muscles cannot be seen in X-ray CT but can be seen in MRI scans. By coating the tympanic membrane with a thin oil layer containing $\mathrm{BaO}_{2}$ (or another substance that absorbs X-radiation well), we were also able to image the shape of the tympanic membrane with X-ray CT.

Preparation and scanning time is only a small fraction of the time required for physical sectioning, which makes it possible to make dedicated models for single experimental temporal bones. The procedure of segmentation is time-consuming but it is expected that highly automated segmentation based on templates, which are currently under development (e.g., Rabbitt et al. 1995; Christensen et al. 1996; Bowdon et al. 1998; Weiss et al. 1998; Woods et al. 1998), could greatly reduce manual intervention in the making of models.

For the interpretation and validation of experimental results, the geometrical data of a 3-D model are of great interest (Decraemer et al. 2002). We have used 3-D models of the middle-ear ossicles for the interpretation and presentation of middle-ear vibration. The construction of a 3-D model of the middleear ossicular chain with good detail takes a few days. When the number of experiments is not too large, it is now possible to have dedicated models for each temporal bone used. In view of the large variability between different ears, this presents a major advantage over using an "average" standard model.

We have also shown how a 3-D model can be used to recover a "loss of reference system." This occurs when an object under study has to be taken out of an experimental setup (either voluntarily to apply a manipulation or a change in orientation or involuntarily due to some unexpected cause) and the object has to be repositioned.

Another important use we have made of imaging of the middle ear was in the design of anatomically correct 3-D models for finite-element simulation.

Physical models of the ear can now be complemented by the distribution of virtual 3-D models on the Internet. Specialized viewer programs enable the user to rotate the structures, to zoom in on details, to make certain structures transparent, or to make crosssections at arbitrarily chosen orientations. Such models can provide a globally available tool for medical students.

Note: Abstracts from ARO meetings mentioned below can be read at the ARO web site: http:// www.aro.org/abstracts/abstracts.html

\section{ACKNOWLEDGMENTS}

We would like to thank H.M. Ladak (The University of Western Ontario, London, Canada) for introducing us to the Iterative Closest Point procedure and for providing us with his Matlab (MathWorks) code implementing the method. We are also indebted to M.M. and O.W. Henson (The University of North Carolina, Chapel Hill, NC, USA) who provided us with MRM data for human, cat, bat, and gerbil temporal bones; and to A.H. Voie (University of Washington, Seattle, WA, USA) who provided us with OPFOS data for the guinea pig. Last but not least, we are very grateful to S.M. Khanna (Columbia University, New York, NY, USA), with whom were performed all of the middle-ear vibration measurements mentioned in this article. This work was supported by the Emil Capita Fund, NOSH, the Fund for Scientific Research (Flanders, Belgium), the Research Funds of the University of Antwerp (RUCA), the Canadian Institutes of Health Research, and the Natural Sciences and Engineering Research Council (Canada).

\section{REFERENCES}

Besl PJ, McKay ND. A method for registration of 3-D shapes, IEEE Trans. Pattern Anal. Machine Intell. 14:239-256, 1992.

Bowden AE, Rabittt RD, Weiss JA. Anatomical registration and segmentation by warping template finite element models. SPIE V 3254:469-476, 1998.

Christensen GE, Rabbitt RD, Miller MI. Deformable anatomical templates using large deformation kinematics. IEEE Trans. Image Processing 5 (10):1435-1447, 1996. 
Decraemer WF, Khanna SM. New insights in the functioning of the middle-ear. In: Rosowski JJ, Merchant S (eds) The function and mechanics of normal, diseased and reconstructed middle-ears. Kugler Publications, The Hague, The Netherlands, 1999, pp 23-38.

Decraemer WF, Khanna SM. Three-dimensional vibration of the ossicular chain in the cat. In: Tomasini EP (ed) Vibration Measurements by Laser Techniques: Advances and Applications. SPIE, Bellingham, WA, Vol 4072, 2000, pp 401-411.

Decraemer WF, Khanna SM, Funnell WRJ. Measurement and modelling of the three-dimensional vibrations of the stapes in cat. In: Wada H, Takasaka T, Ikeda K, Ohyama K, Koike T (eds) Proceedings of the Symposium on Recent Developments in Auditory Mechanics. World Scientific Publishing, River Edge, NJ, 2000, pp 36-43.

Decraemer WF, Khanna SM, Dirckx JJJ. The integration of detailed 3-dimensional anatomical data for the quantitative description of 3-dimensional vibration of a biological structure. An illustration from the middle-ear. In: Tomasini EP (ed) Vibration Measurements by Laser Techniques: Advances and Applications. SPIE, Bellingham, WA, Vol 4872, 2002, pp 148158.

FUNNELL WRJ. On the choice of a cost function for the reconstruction of surfaces by triangulation between contours. Comp. Struct. 18 (1):23-26, 1984.

FunNELl SM, FunNell WRJ (1988) An approach to finite- element modelling of the middle ear. Proceeding of the 14th Canadian Medical and Biological Engineering Conference. Canadian Medical and Biological Engineering Society, Orleans, Ontario, Canada, pp 101-102.

Funnell WRJ, Decraemer WF, Khanna SM. On the rigidity of the manubrium in a finite-element model of the cat eardrum. J. Acoust. Soc. Am. 91 (4):2082-2090, 1992.

FUNNELL WRJ. Finite-element modelling of the cat middle-ear with elastically suspended malleus and incus. Assoc. Res. Otolaryngol. Abs. 779, 1996.

FunNELL WRJ (2002) http://funsan.biomed.mcgill.ca/ funnell/ AudiLab/sw/fie.html

HaAcke EM, Brown RW, Thompson MR, Venkatesan R. Magnetic resonance imaging. Physical principles and sequence design. Wiley-Liss, New York, 1999.

Henson MM, Henson Jr OW, Gewalt SL, Wilson JL, Johnson GA. Imaging the cochlea by magnetic resonance microscopy. Hear. Res. 75:75-80, 1994.

Herman GT. Image Reconstruction from Projections. The fundamentals of computerized tomography. Academic Press, New York, 1980.

Kak AC, Slaney M. Principles of computerized tomographic imaging. IEEE Press, New York, 1988,

Kass M, Witkin A, Terzopoulos D. Snakes: Active contour models. Int. J. Comput. Vision. 1:321-331, 1987.

Kuperman V. Magnetic resonance imaging. Principles and applications. Academic Press, Orlando, FL, 2000,

Kuypers LC, Decraemer WF, Dirckx JJJ. Thickness measurements of a cat's eardrum by use of a confocal microscope. Assoc. Res. Otolaryngol. Abs. 376, 2001.

LoRensen WE, Cline HE. Marching cubes: A high resolution 3D surface construction algorithm. Comput. Graph. (ACM SIGGRAPH) 21 (4):163-169, 1987.

Mason TP. Virtual temporal bone. Otolaryngol. Head Neck Surg. 122 (2):168-173, 2000.
McInerney T, Terzopoulus D. Deformable models in medical image analysis. Med. Image Anal. 1 (2):91-108, 1996.

Nomura Y, Okuno T, Hara M, Shinagawa Y, Kuni TL. Walking through a human ear. Acta Otolaryngol. 107 (5-6):366-370, 1989.

Northrop C, Levine S, Collin S. 3D video model of the middle-ear - A study of the patterns of aeration. Assoc. Res. Otolaryngol. Abs. 370, 2001.

Pfister H, Lorensen We, Bajaj C, Kindlmann G, Schroeder W, Avila LS, Raghu KM, Machiraju R, Lee J. The transfer function bake-off. IEEE Comput. Graph. Appl. 21 (3):16-22, 2001.

Rabbitt RD, Weiss J, Christensen G, Miller MI. Mapping of hyperelastic deformable templates using the finite element method. SPIE V 2573:252-265, 1995.

Roвb RA (1995) Three-Dimensional Biomedical Imaging. VCh Publishers, Inc., New York

Sasov A, VAn Dyck D. Desktop x-ray microscopy and microtomography. J. Microsc. 191:151-158, 1998.

SijBers J, Scheunders P. Watershed-based segmentation of 3D MR data for volume quantizaton. Magn. Res. Imaging 15 (6):697688, 1997.

Vincent L, Sollle V. Watershed in digital spaces: an efficient algorithm based on immersion simulations, IEEE Trans. Pattern Anal. Machine Intell. 13 (6):538-598, 1991.

Vogel U, Sмiтt T. 3D-visualization of middle-ear structures. Image Display. Proc. SPIE Med. Imaging 3335:141-151, 1998.

VogEL U. 3D-imaging of internal temporal bone structures for geometric modelling of the human hearing organ. In: Wada $\mathrm{H}$, Takasaka T, Ikeda K, Ohyama K, Koike T (eds) Proceedings of the Symposium on Recent Developments in Auditory Mechanics. World Scientific Publishing, River Edge, NJ, 2000, pp 44-50.

Voie AH, Burns DH, Spelman FA. Orthogonal-plane fluorescence optical sectioning: three-dimensional imaging of biological specimens. J. Microsc. 170 (3):229-236, 1993.

VOIE FA. Three-dimensional reconstruction and quantitative analysis of the mammalian cochlea. Ph.D. Thesis, University of Washington, 1996.

Voie AH, Spelman FA. Three-dimensional reconstruction of the cochlea from two-dimensional images of optical sections. Comput. Med. Imaging Graph. 19 (5):337-384, 1996.

VoIE AH. Whole-specimen laser imaging of the intact guinea pig cochlea within the tympanic bulla. Assoc. Res. Otolaryngol. Abs. 875, 2001.

VoIE AH. Microchannels in the modiolar wall of the human scala tympani. Assoc. Res. Otolaryngol. Abs. 380, 2002a.

VoIE AH. Three-dimensional computer reconstruction and quantitative analysis of the basal region of the scala tympani in the human cochlea. Assoc. Res. Otolaryngol. Abs. 381, 2002b.

WARRICK PA, FUNNELL WRJ. A VRML based anatomical visualization tool for medical education. IEEE Trans. Inform. Technol. Biomed. 2 (2):55-61, 1998.

Weiss JA, Rabbitt RD, Bowden AE, Maker BN. Incorporation of medical image data in finite element models to track strain in soft tissues. SPIE V 3254:477-476, 1998.

Woods RP, Grafton ST, Holmes CJ, Cherry SR, Mazziotta JC. Automated image registration: I. General methods and intrasubject, intramodality validation. J. Comput. Assisted Tomogr. 22:139-152, 1998.

Xu C, Prince JL. Gradient vector flow: a new external force for snakes. IEEE Proc. Conf. Comp. Visual Pattern Recog. CVPR'97) 66-71, 1997. 